\title{
Sistemas Termodinámicos
}

\author{
Thermodynamic Systems
Julio C. Gil-Atanacio ${ }^{a}$, Luis A. Villeda-Cruz ${ }^{b}$, Daniel Pérez-Segovia ${ }^{c}$, Juan C. Mucio- Martínez $^{d}$

\begin{abstract}
:
A thermodynamic system is defined as the part of the universe understudy. The thermodynamic system can be defined by real or imaginary walls, it contains what is called an object of study, this thermodynamic system can undergo internal transformations and exchange energy and / or matter with the external environment. The system is s eparated from the environment by the system limit, the limit can be fixed or mobile, there are also types of systems in which we can find, the open system, closed system, isolated system, each of these have certain characteristics to that can be easily identified. The purpose of this research work is that the student or the reader can get an idea in the interpretation of the presented topic, and thus be able to contribute to their knowledge about the topic.
\end{abstract}

\section{Keywords:}

Open system, closed system, isolated system, bordert

\begin{abstract}
Resumen:
Un sistema termodinámico se define como la parte del universo objeto de estudio. El sistema termodinámico puede estar definido por paredes reales o imaginarias, contiene lo que se llama un objeto de estudio, este sistema termodinámico puede experimentar transformaciones internas e intercambiar energía y/o materia con el entorno externo. El sistema está separado del entorno por el límite del sistema, el límite puede ser fijo o móvil, además existen tipos de sistemas en los que podemos encontrar, el sistema abierto, sistema cerrado, sistema aislado, cada uno de estos cuentan con ciertas características para que se puedan identificar fácilmente. La finalidad de este trabajo de investigación es que el alumno o el lector pueda obtener una idea en la interpretación del tema presentado, y así poder contribuir en su conocimiento acerca del tema.
\end{abstract}

\section{Palabras Clave:}

Sistema abierto, sistema cerrado, sistema aislado, frontera

\section{Introducción}

Un sistema es la parte del universo que se estudia. Un sistema termodinámico debe ser suficientemente grande, es decir debe poseer un gran número de partículas (o gran número de grados de libertad) [1]. Los sistemas que consisten en un número reducido de partículas (o que tienen pocos grados de libertad) nunca constituirán un sistema termodinámico [1].
Los sistemas termodinámicos se clasifican según la transferencia de masa y de energía a través de las fronteras del sistema y el efecto que esas interacciones ejercen sobre el estado del sistema [2]. Según lo anterior, se clasifican en sistemas abiertos, cerrados y aislados.

\section{Sistema cerrado}

Es un sistema termodinámico donde hay intercambio de energía con el resto del universo, pero sin haber un

\footnotetext{
a Autor de correspondencia, Universidad Autónoma del Estado de Hidalgo, https://orcid.org/0000-0003-2882-5234, Email: gi415567@uaeh.edu.mx

b Universidad Autónoma del Estado de Hidalgo, https://orcid.org/0000-0002-2297-9171, Email: vi414535 @uaeh.edu.mx

c Universidad Autónoma del Estado de Hidalgo, https://orcid.org/0000-0003-4302-7038, Email: pe413284@uaeh.edu.mx

dUniversidad Autónoma del Estado de Hidalgo,https://orcid.org/0000-0001-6119-6586, Email: mu414201@uaeh.edu.mx
} 
intercambio físico de materia con el universo esto quiere decir que la materia que se encuentre dentro del sistema podrá cambar su temperatura dependiendo del entorno, así como su volumen, pero su peso se no sufrirá ningún cambio. Ninguna masa puede entrar o abandonar un sistema cerrado.

Aunque en un sistema cerrado la cantidad de materia es fija (constante), se permite a la energía traspasar sus límites en forma de trabajo y calor.

El volumen de un sistema cerrado no tiene que ser fijo.

La materia también puede cambiar de composición química dentro del sistema La cantidad de materia bajo observación suele denominarse masa [4]

Ejemplos de sistemas cerrados:

- Un reloj a cuerda, que para su funcionamiento necesita que no exista modificación por la temperatura o el medio externo.

- Un termo perfectamente construido para que la temperatura se conserve sin cambios en lo más mínimo.

- El planeta tierra (intercambia energía, pero no materia).

\section{Sistema abierto}

Es un sistema que interactúa continuamente con su entorno. Un sistema abierto es lo contrario al sistema aislado ya que no intercambia energía, materia o información con el medio ambiente. Todos o casi todos los sistemas naturales están abiertos.

En los sistemas termodinámicos, el sistema abierto no puede existir en el estado de equilibrio puesto que hay un intercambio de energía y materia entre el sistema y los alrededores.

En muchos casos es mejor pensar en término de una región dada del espacio a través de la cual fluye la masa. Esta región se denomina también volumen de control.

El flujo a través de estos dispositivos se estudia mejor al seleccionar la región dentro del dispositivo como el volumen de control.

Tanto masa puede cruzar la frontera de un volumen de control, así como también la energía puede hacerlo en forma de trabajo y calor.

El término volumen o masa de control también se usa en un sistema cerrado.

El término sistema abierto se usa intercambiablemente con el de volumen de control.

Cuando se usan los términos masa de control o volumen de control, el contorno del sistema recibe el nombre de superficie de control [3].

Ejemplos de sistemas abiertos:

- La célula, pues cuenta con una membrana semipermeable que produce el intercambio con el exterior.
- Una planta, que en el proceso de fotosíntesis realiza un notorio intercambio de energía.

- Un curso de agua como un río, que recibe afluentes y envía a otros cursos.

- Cada uno de los órganos o sistemas del cuerpo humano puede interpretarse como un sistema abierto.

\section{Sistema aislado}

Un sistema termodinámico aislado es un sistema que no puede interactuar con el resto del universo debido a que su frontera es impermeable tanto a la materia como a la energía. Un sistema aislado es, por lo tanto, un tipo especial de sistema cerrado cuya frontera es adiabática y además es rígida e impermeable a los campos de fuerzas. Lo importante del concepto del sistema termodinámico aislado no es que haya o no sistemas perfectamente aislados, sino que es posible considerar muchos sistemas como aislados a efectos prácticos: los efectos que delatan que no están aislados son de escasa importancia en el fenómeno que se está estudiando [2]. Ejemplos de sistemas aislados:

- Una bombona de gas, contenido a presión en su interior, el gas está aislado de la materia y la energía a su alrededoren condiciones normales.

- Los alimentos enlatados, en condiciones normales, estos alimentos se encuentran lejos de cualquier intercambio de materia 0 de energía.

- Una caja fuerte, el contenido en las cajas fuertes está separado por gruesas capas herméticas de metal de su entorno, aislado de la materia y de la energía,

- Los trajes de neopreno, un hombre embutido en estos trajes, usualmente para el buceo o submarinismo, se encuentra protegido durante un período de tiempo del intercambio calórico entre el agua y su cuerpo.

\section{Fronteras}

El sistema termodinámico puede estar separado del resto del universo (denominado alrededores del sistema) por paredes reales o imaginarias. Las paredes que separan un sistema de sus alrededores pueden ser aislantes (Ilamadas paredes adiabáticas) o permitir el flujo de calor (diatérmicas). [3]

La frontera de un sistema puede ser:

a) Fija (las paredes de un recipiente) o móvil (un émbolo o pistón de un motor de explosión).

b) Permeable a la masa o impermeable a ella. En el primer caso se dice que tenemos un sistema abierto (p.ej. un motor en el que entra combustible por un lado y salen gases por otro) $y$ en el segundo uno cerrado (p.ej. en el circuito 
de refrigeración de una nevera, el gas freón que circula por los tubos nunca sale al exterior).

c) Permeable al calor o impermeable a él. Si al poner en contacto el sistema con el ambiente se produce una transferencia de energía debido a la diferencia de temperaturas, se dice que la frontera es diatermia. Si el calor no puede atravesar la frontera se dice que ésta es adiabática. [5]

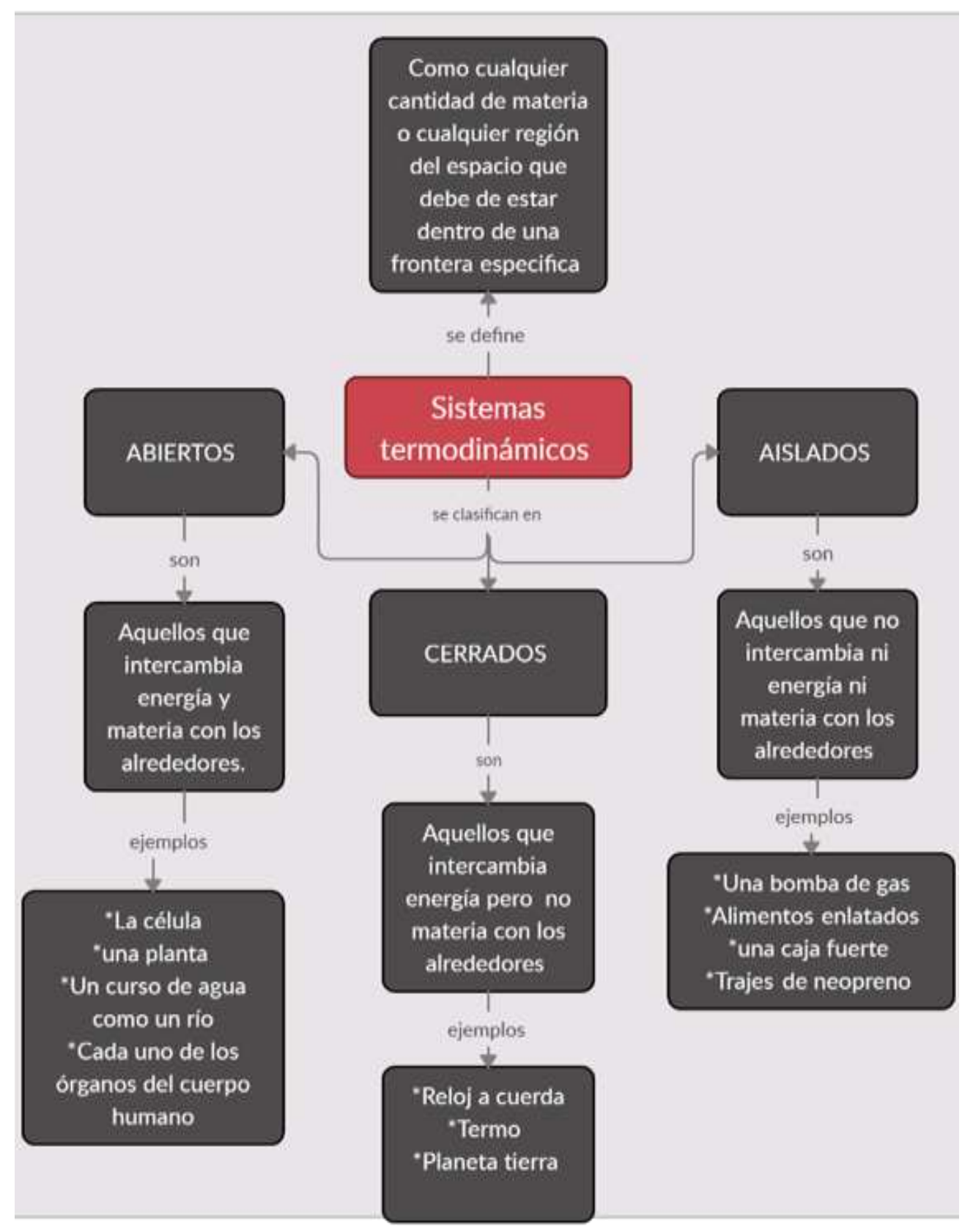




\section{Agradecimientos}

Gracias a UAEH por brindarnos el apoyo y confianza que se nos ha dado en todo momento. De igual manera agradecemos a la Dra. Lizeth Martínez Ayala que nos brindó parte de su tiempo para asesorarnos y brindamos el apoyo, su experiencia y conocimientos para poder concluir con este tema.

\section{Referencias}

[1] Fernández, M. D. C. L. (2001). Termodinámica (Vol. 53). Universidad de Sevilla, pp. 10-19.

[2] Oriol Planas. Sistema termodinámico, tipos de sistemas y definición. (2020), De ingeniero técnico, pp. 1-2.

[3] Rolle, K. C. (2006). Termodinámica. Pearson Educación. Pearson educación, México, 2006, pp. 34-40.

[4] Sears, F.W., \& Salinger, G.L. (1978). Termodinámica teoría cinética y termodinámica estadística. Reverte, pp. 1-5.

[5] Zemasnsky, M.W, \& Dittman, R.H. (1997). Calor y Termodinámica (Vol.18). Aguilar. Ciudad de México, pp. 2426.

[6] Dugan, R.E., \& Jones, J.B. (1996). Ingeniería termodinámica. Prentice Hall, pp. 11-17. 